\title{
Ein simulationsbasiertes Optimierungssystem zur Priorisierung von Maschinenstillständen unter Einbeziehung eines Lookahead
}

\author{
Michael Hegemann ${ }^{1 *}$, Stefan Nickel ${ }^{2}$ \\ ${ }^{1}$ Mercedes-Benz AG, Mercedesstraße 120, 70372 Stuttgart, Deutschland; *michael.hegemann@daimler.com \\ ${ }^{2}$ Institut für Operations Research, Diskrete Optimierung und Logistik, Karlsruher Institut für Technologie (KIT), \\ Karlsruhe, Deutschland
}

\begin{abstract}
This paper discusses the added value of prioritizing machine breakdowns, taking into account future planned downtime. For this purpose, an online-optimization problem with lookahead is formulated, whereby the information about planned downtimes is available as lookahead. To solve the problem a simulation-based optimization system is presented. By implementing this system in a simulation environment and performing a simulation study of a multidimensional flow production system it is shown that a prioritization with lookahead can reduce the occurrence of momentary bottlenecks due to planned downtimes, so that the overall performance of a production system can be increased.
\end{abstract}

\section{Einleitung}

Während der geplanten Betriebszeit eintretende Maschinenstillstände, resultierend beispielsweise aus technischen Störungen oder Wartungsmaßnahmen, führen bei hoch ausgelasteten Produktionssystemen zu hohen Produktionsverlusten [1]. Dabei besteht die Möglichkeit, dass zeitgleich Stillstände an mehreren Produktionsressourcen eines Produktionssystems anliegen. Da in der Praxis die Anzahl der Produktionsmitarbeiter, die zur Stillstandsbehebung zur Verfügung stehen, oftmals begrenzt ist, können durch eine objektive Priorisierung der Stillstände nach ihrer jeweiligen Auswirkung auf das Produktionssystem die Reaktionszeiten auf die schwerwiegendsten Stillstände reduziert und Produktionsverluste nachhaltig minimiert werden [2].

Neben den zu priorisierenden ungeplanten Stillständen können zum Zeitpunkt der Priorisierung auch Informationen über zukünftig eintretende geplante Stillstandszeiten vorliegen. Diese Stillstandszeiten resultieren beispielsweise aus anstehenden präventiven Instandhaltungsmaßnahmen. Zusätzliche Informationen über geplante Stillstandszeiten ergeben sich aus der Anwendung von Predictive Maintenance bzw. einer zustandsorientierten, vorausschauenden Instandhaltungsstrategie [3].
In diesem Kontext konnte bereits für verkettete Fließfertigungssysteme gezeigt werden, dass eine Berücksichtigung zukünftig eintretender geplanter Stillstandszeiten zu einer anderen Priorisierungsreihenfolge führen kann [4]. In diesem Beitrag wird darauf aufbauend ein operatives Entscheidungsunterstützungssystem vorgestellt, welches den Produktionsmitarbeitern eine objektiv ermittelte Priorisierungsreihenfolge der zum Entscheidungszeitpunkt anliegenden Stillstände zur Verfügung stellt. Bei der Ermittlung der Priorisierungsreihenfolge wird dabei nicht nur die Auswirkung der in diesem Moment anliegenden Stillstände berücksichtigt, sondern es werden auch zukünftig eintretende geplante Stillstandszeiten in Form eines Lookahead als zusätzliche Information in die Entscheidungsfindung mit einbezogen. Damit soll erreicht werden, dass auch die Auswirkungen geplanter Stillstände auf die Produktionsleistung eines Fließfertigungssystems minimiert und so Produktionsverluste nachhaltig reduziert werden.

Nach einem kurzen Überblick über den aktuellen Stand der Technik wird eine simulationsbasierte Vorgehensweise vorgestellt, mit der die Priorisierung von Maschinenstillständen unter Berücksichtigung eines Lookahead möglich ist. Ferner wird die Implementierung dieser Vorgehensweise im Rahmen eines Entscheidungsunterstützungssystems in eine Simulationsumgebung skizziert. Abschließend werden die Ergebnisse einer durchgeführten Simulationsstudie zur Untersuchung des erzielbaren Mehrwerts einer Priorisierung mit Lookahead dargestellt.

\section{Stand der Technik}

In der Praxis wird für die Priorisierung ungeplanter Stillstände häufig ein subjektives Verfahren, basierend auf Expertenerfahrung, Expertenwissen oder Intuition, angewendet [5]. 
Bei wissenschaftlichen Ansätzen kommen hingegen für die Ermittlung einer Priorisierungsreihenfolge vor allem statische Verfahren, bei denen die Priorisierung anhand einer zuvor festgelegten Reihenfolge erfolgt, oder dynamische Verfahren, die überwiegend auf der Ermittlung eines Engpassrankings basieren, zur Anwendung. So werden in [6] und [7] zwei statische Bewertungsschemata vorgestellt, anhand derer Maschinen entsprechend ihrer Kritikalität für das Produktionssystem priorisiert werden können. Weitere statische Verfahren basieren auf der Bestimmung eines Engpassrankings. Die Priorisierung erfolgt dabei mittels eines bereits vor dem Entscheidungszeitpunkt feststehenden Rankings. Zur Engpassermittlung werden Methoden wie die „Active Period Method“ [8] [9], die „Shifting Bottleneck Detection Method“ [10], die „Arrow Based Method“ [11] sowie die „Turning Point Method“ [12] [13] angewendet.

Im Gegensatz zu statischen Verfahren wird bei dynamischen Verfahren die Priorisierungsreihenfolge jeweils zum Entscheidungszeitpunkt auf Grundlage des aktuellen Systemzustandes neu bestimmt. In [14] und [15] wurde dazu eine simulationsbasierte Priorisierungsmethode hergeleitet, mit der basierend auf Online-Produktionsinformationen eine Priorisierungsreihenfolge aktuell anliegender Stillstände zum jeweiligen Entscheidungszeitpunkt ermittelt werden kann. Im Gegensatz dazu wird in [16] eine analytische Engpassermittlungsmethode, die „Shifting Bottleneck Detection Method“, verwendet, um auf Basis von Echtzeitproduktionsdaten die momentanen Engpässe zu bestimmen und anhand derer eine Priorisierung von Maschinenstillständen vorzunehmen. In [2] wurde zudem eine Priorisierungsmethode hergeleitet, die auf der Bestimmung von Echtzeit-Engpässen und Engpässen in naher Zukunft beruht.

Die zur Bestimmung der Priorisierungsreihenfolge verwendeten Daten beziehen sich dabei häufig auf einen historischen Zeitraum oder aber auf den aktuellen Zustand des Produktionssystems. Einen Schritt weiter wird in [17] gegangen, indem mittels historischer Produktionsdaten die Engpässe der nächsten Zeitperiode prognostiziert werden, wobei hier bereits angemerkt wird, dass diese prognostizierten Engpässe letztendlich auch in die Priorisierungsentscheidung mit einfließen sollten.

Zusammenfassend zeigt die Analyse wissenschaftlicher Ansätze, dass bisher kein Ansatz existiert, der zum Entscheidungszeitpunkt verfügbare Informationen über zukünftig eintretende geplante Stillstandszeiten konkret in die Priorisierungsentscheidung ungeplanter Maschinenstillstände miteinbezieht.

\section{Priorisierung mit Lookahead}

Im Folgenden wird eine simulationsbasierte Vorgehensweise vorgestellt, mit der die Ermittlung einer Priorisierungsreihenfolge unter Berücksichtigung zukünftig eintretender geplanter Stillstandszeiten möglich ist.

Der Ausgangspunkt ist ein Entscheidungszeitpunkt $t_{d}$, an dem ein Produktionsmitarbeiter entscheiden muss, welcher der zu diesem Zeitpunkt anliegenden Stillstände als Nächstes instandgesetzt wird. Damit eine Priorisierung überhaupt notwendig ist, muss dabei die Anzahl zu priorisierender Stillstände größer als eins sein. Ist die Anzahl eins, so liegt nur ein Stillstand an und der Mitarbeiter kann direkt zugeordnet werden.

Liegen mehrere Stillstände an, wird eine Priorisierungsreihenfolge bestimmt. Um die Priorisierungsreihenfolge ungeplanter Stillstände unter Berücksichtigung zukünftig eintretender geplanter Stillstandszeiten zu ermitteln, können die zu priorisierenden Stillstände unter der Annahme, dass zeitlich parallel anliegende Stillstände nur sequentiell behoben werden, in ein Reihenfolgenoptimierungsproblem transformiert werden [14]. Die Formulierung dieses Optimierungsproblems ergibt sich mit der zu maximierenden Zielgröße Durchsatzmenge der Engpassprozessstufe wie folgt:

$$
\text { Max } \rightarrow \text { Durchsatzmenge }=\max _{k=1}^{L} D\left(\operatorname{Seq}_{k}\right)
$$

Dabei sind $L$ die Anzahl möglicher Priorisierungsreihenfolgen und $D\left(S e q_{k}\right)$ die mit der Reihenfolge $k$ zu einem Vergleichszeitpunkt $T_{E n d e}$ erzielbare Durchsatzmenge der statischen Engpassprozessstufe. Da der Engpass die Ausbringung des gesamten Produktionssystems beeinflusst, wirken sich die unterschiedlichen Behebungsreihenfolgen ebenfalls auf die Durchsatzmenge des Engpasses aus. Als statische Engpassprozessstufe für das Abbild eines Fließfertigungssystems zum Zeitpunkt $t_{d}$ wird hier die Prozessstufe mit der größten Taktzeit definiert. Diese limitiert die Ausbringung des gesamten Fließfertigungssystems, solange keine Stillstände anliegen, die zu einem Wartezustand der statischen Engpassprozessstufe aufgrund eines Werkstückmangels am Einlauf oder eines blockierten Auslaufs führen. Sollten mehrere Prozessstufen dieselbe Taktzeit aufweisen, so ist die am weitesten stromabwärtsgelegene Stufe die Engpassprozessstufe. Um die Engpassprozessstufe auch für mehrdimensionale Fließfertigungssysteme zu ermitteln, kann mit folgender Formel eine repräsentative Taktzeit für die insgesamt $n$ redundant angeordneten Prozesse einer redundanten Prozessstufe, die in der Praxis häufig 
auch als Parallelmaschine bezeichnet wird, berechnet werden [2]:

$$
T Z_{\text {rep }}=\left[\sum_{i=1}^{n} \frac{1}{T Z_{i}}\right]^{-1}
$$

Da sich die Zielfunktion für komplexe Fließfertigungssysteme meistens nicht in geschlossener Form darstellen lässt, wird die ereignisdiskrete Simulation zur Bestimmung des Zielfunktionswertes eingesetzt. Dieses simulationsbasierte Optimierungssystem, bei dem die dominierende Komponente die Optimierung ist, welche auf das Simulationsmodell als Zielfunktionswert zurückgreift und als Ergebnis die erzielbare Durchsatzmenge der untersuchten Behebungsreihenfolge zurückgegeben bekommt, ist in Abbildung 1 dargestellt.

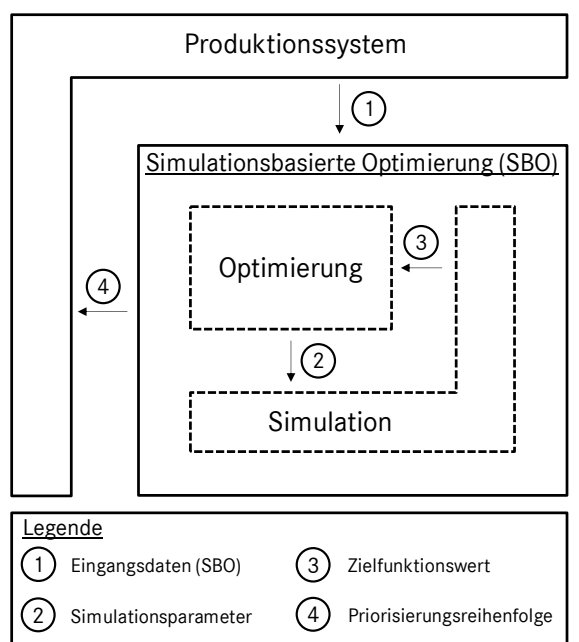

Abbildung 1: Schematische Darstellung des SBO-Systems

Um zukünftige geplante Stillstandszeiten bei der Priorisierung von Maschinenstillständen zu berücksichtigen, wird das vorliegende Optimierungsproblem als Online-Optimierungsproblem mit Lookahead aufgefasst. Im Gegensatz zur Offline-Optimierung, bei der zu Beginn alle Eingabedaten bekannt sind, werden bei der OnlineOptimierung die Eingabedaten sequentiell bekannt gegeben, d. h. bei einer Online-Optimierung müssen Entscheidungen unmittelbar und ohne Wissen über zukünftige Ereignisse getroffen werden [18]. Bei einer Online-Optimierung mit Lookahead ist nun eine Teilmenge der zukünftigen Eingabedaten bekannt, sodass mehr Informationen für die Entscheidung bereitstehen. Somit kann die Online-Optimierung mit Lookahead zwischen den Extrema der klassischen Offline-Optimierung und der reinen Online-Optimierung eingeordnet werden [19]. In diesem Kontext können geplante Stillstandszeiten, die zum Zeitpunkt der Priorisierung bereits bekannt sind, als
Lookahead klassifiziert werden. Dadurch ist es möglich, die im Lookahead enthaltenen geplanten Stillstandszeiten in den Simulationsläufen zur Ermittlung der Zielfunktionswerte der potenziellen Priorisierungsreihenfolgen zu berücksichtigen und so letztendlich eine Priorisierung unter Einbeziehung zukünftig eintretender geplanter Stillstände zu ermöglichen.

Der Vergleichszeitpunkt, der im Folgenden als Simulationsendzeitpunkt $T_{\text {Ende }}$ bezeichnet wird und zu dem die Ergebnisgrößen für die Bewertung der Behebungsreihenfolgen bestimmt werden, ist dadurch charakterisiert, dass sich die Auswirkungen der im Lookahead enthaltenden Stillstandszeiten vollständig in der Durchsatzmenge der statischen Engpassprozessstufe realisiert haben. Um den Simulationsendzeitpunkt bereits zum Entscheidungszeitpunkt $t_{d}$ zu bestimmen, wird derjenige Stillstand aus dem Lookahead verwendet, dessen Summe aus Stillstandseintrittszeitpunkt $T_{\text {Start }}$ und geplanter Stillstandsdauer TTR am weitesten in der Zukunft liegt. Der Simulationsendzeitpunkt ergibt sich dann mit der Nachwirkzeit $\Delta t_{\text {Nachwirkzeit }}$ sowie der Prozesszeit der statischen Engpassprozessstufe $P Z_{E n g}$ wie folgt:

$$
T_{\text {Ende }}=T_{\text {Start }}+T T R+\Delta t_{\text {Nachwirkzeit }}+P Z_{\text {Eng }}
$$

Für einen Prozess der sich stromaufwärts der statischen Engpassprozessstufe befindet, ist die Nachwirkzeit die Zeitdauer, bis das erste Werkstück nach Stillstandsende am Einlauf der Engpassprozessstufe zur Verfügung steht und entspricht damit der Durchlaufzeit vom Einlauf des Prozesses bis zum Einlauf der Engpassprozessstufe. Befindet sich der Prozess hingegen stromabwärts der statischen Engpassprozessstufe, so ist die Nachwirkzeit die Zeitdauer, bis die Engpassprozessstufe das erste Werkstück nach Stillstandsbehebung an die nachfolgende Prozessstufe weitergeben und damit wieder entsprechend ihrer Taktzeit Werkstücke bearbeiten kann.

Nachdem die statische Engpassprozessstufe und der Simulationsendzeitpunkt bestimmt wurden, kann für jede Behebungsreihenfolge $k$ ein Simulationslauf durchgeführt werden. Dazu wird der Zustand des Fließfertigungssystems zum Entscheidungszeitpunkt $t_{d}$ als Startbedingung für das Simulationsmodell verwendet, sodass das Simulationsmodell ein möglichst genaues Abbild des realen Fließfertigungssystems ist. Auf die für die Initialisierung verwendeten Daten wird im Rahmen der Implementierung nochmals näher eingegangen. 
Die zu priorisierenden Prozesse stehen zu Beginn eines Simulationslaufes still und werden im Simulationsverlauf entsprechend der zu bewertenden Behebungsreihenfolge sequentiell wieder produktiv geschaltet. Um die zukünftig eintretenden geplanten Stillstandszeiten zu berücksichtigen, werden die im Lookahead enthaltenden Stillstände für jeden Simulationslauf so parametrisiert, dass die betroffenen Prozesse entsprechend dem jeweiligen Eintrittszeitpunkt und der geplanten Stillstandsdauer stillstehen. Da neben den zu priorisierenden Stillständen und den zukünftig eintretenden geplanten Stillstandszeiten keine weiteren Stillstände und auch keine anderen stochastischen Einflüsse, wie beispielsweise Taktzeitschwankungen, während des Simulationslaufs berücksichtigt werden, liegt hier folglich ein deterministisches Modell vor. Ein Simulationslauf endet, sobald der Simulationsendzeitpunkt $T_{\text {Ende }}$ erreicht ist. Zum Zeitpunkt $T_{\text {Ende }}$ werden die Durchsatzmenge und die Restprozesszeit der statischen Engpassprozessstufe ausgelesen und zwischengespeichert.

Sind schließlich alle $L$ Behebungsreihenfolgen simuliert, kann die Priorisierungsreihenfolge ermittelt werden. Diese entspricht nach Formel 1 der Behebungsreihenfolge mit dem größten Wert für die Durchsatzmenge der Engpassprozessstufe. Tritt der Fall ein, dass mehrere Behebungsreihenfolgen den maximalen Wert aufweisen, so wird als zweites Entscheidungskriterium die Restprozesszeit zum Zeitpunkt $T_{\text {Ende }}$ verwendet, wobei die Behebungsreihenfolge mit der geringsten Restprozesszeit die Priorisierungsreihenfolge darstellt. Sollte es weiterhin mehr als eine Reihenfolge geben, die die maximale Durchsatzmenge und die minimale Restprozesszeit aufweisen, sind diese Reihenfolgen im Sinne der Auswirkung auf die Ausbringung des Fließfertigungssystems identisch. Tritt dieser Fall ein, so kann eine definierte Auswahl mittels einer FCFS-Regel getroffen werden, wobei die Priorisierungsreihenfolge die Reihenfolge ist, deren am höchsten priorisierter Stillstand zeitlich am frühsten eingetreten ist.

\section{Implementierung in Plant Simulation}

Um den Mehrwert einer Priorisierung ungeplanter Stillstände unter Berücksichtigung zukünftig eintretender geplanter Stillstandszeiten näher zu untersuchen, wurde die im vorherigen Kapitel beschriebene simulati- onsbasierte Vorgehensweise zur Priorisierung von Stillständen mit Lookahead in Form eines Entscheidungsunterstützungssystems in eine Simulationsumgebung (Siemens Plant Simulation) umgesetzt. Das Entscheidungsunterstützungssystem besteht dabei aus zwei Instanzen, die über die Plant Simulation eigenen Schnittstellen miteinander kommunizieren. Unter einer Instanz wird hier eine Modelldatei der Simulationssoftware verstanden.

In einer ersten Instanz wird das ablauffähige Modell des untersuchten Fließfertigungssystems und die Ressource Produktionspersonal abgebildet (Abbildung 2).
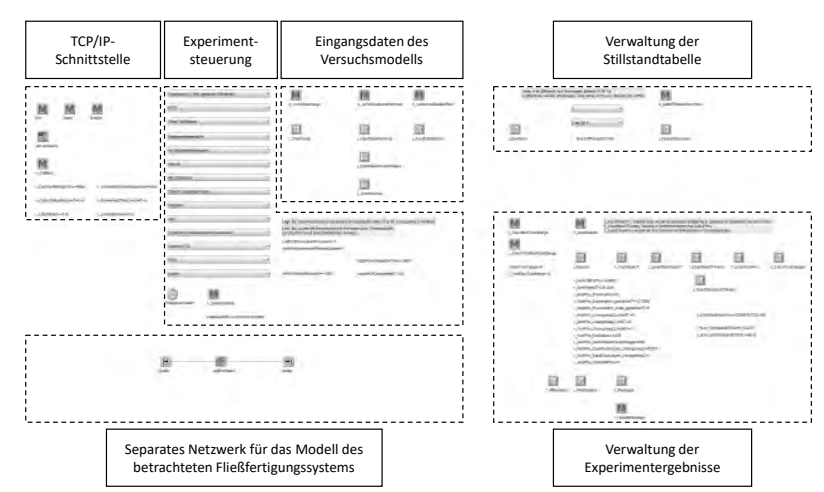

Abbildung 2: Erste Instanz des Entscheidungsunterstützungssystems (Screenshot)

Dieses Modell bildet das dynamische Verhalten des Fließfertigungssystems ab, wobei im Kontext einer Priorisierung von Stillständen die Aufprägung von Stillständen sowie eine entsprechende Steuerung der Stillstandsbehebung entscheidend sind. Dafür wurde anstelle des softwareeigenen Störgenerators eine manuelle Aufprägung mittels einer Stillstandstabelle, in der sämtliche Stillstandszeiten mit Eintrittszeitpunkt und Stillstandsdauer aufgelistet sind, und einem Baustein, der für jeden Produktionsprozess die Einplanung des nächsten Stillstandes in die Ereignisliste übernimmt, umgesetzt. Die Stillstandstabelle wurde in einer separaten Instanz, die ein identisches Simulationsmodells des Fließfertigungssystems beinhaltet, erstellt, indem sämtliche Stillstände mitgeschrieben wurden, die während eines Simulationslaufes aufgetreten sind. Die Stillstände wurden dabei durch den softwareeigenen Störgenerator mit Hilfe der für das Simulationsexperiment verwendeten Werte für die Verfügbarkeiten und die Reparaturdauern erzeugt. Da auf eine Abbildung des Produktionspersonals in diesem Modell verzichtet wird, werden die Stillstände hier umgehend und ohne Wartezeit auf verfügbares Produktionspersonal behoben. Diese manuelle Aufprägung der Still- 
stände hat schließlich den Vorteil, dass sämtliche Stillstandszeiten in einer Tabelle aufgeführt und somit zu jedem Simulationszeitpunkt bekannt sind. Neben der Verwendung exakter Reparaturdauern für die Ermittlung der Priorisierungsreihenfolgen im Rahmen der in diesem Beitrag vorgestellten Simulationsstudie, kann dadurch außerdem die Information über im weiteren Simulationsverlauf eintretende Stillstandszeiten für einen Lookahead generiert werden.

Zur Abbildung des Produktionspersonals wurde das in Plant Simulation verfügbare Konzept einer Vermittlung von Dienste-Angebot und Dienste-Nachfrage mittels eines Broker-Bausteins verwendet. Die Vermittlung der Dienste des Broker-Bausteins wurde dabei problemspezifisch programmiert, sodass die Zuordnung des Produktionspersonals entsprechend der zum jeweiligen Entscheidungszeitpunkt bestimmten Priorisierungsreihenfolge erfolgen kann.

Tritt nun während eines Simulationslaufes der Fall ein, dass die Anzahl an Stillständen größer als die Anzahl zur Verfügung stehender Produktionsmitarbeiter ist, so wird ein Abbild des Produktionssystems durch die Initialisierung eines zweiten Modells (Forecast-Modell) in einer weiteren Instanz erzeugt, wobei in diesem Modell auf eine Abbildung des Produktionspersonals verzichtet wird (Abbildung 3).

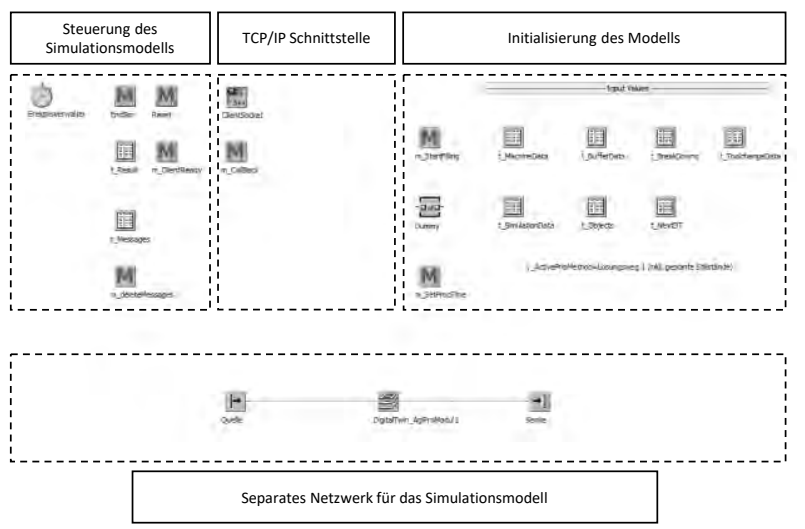

Abbildung 3: Forecast-Modell des Entscheidungsunterstützungssystems (Screenshot)

Mit Hilfe dieses Abbilds, welches den aktuellen Systemzustand des Fließfertigungssystems zum Zeitpunkt der Priorisierung wiederspiegelt, werden dann die Simulationsläufe zur Bestimmung der Durchsatzmenge sowie der Restprozesszeit der Engpassprozessstufe durchgeführt.

Damit zum Entscheidungszeitpunkt der Zustand des Forecast-Modells dem aktuellen Zustand des Modells des Fließfertigungssystems entspricht, wird für jede Priorisierungsentscheidung eine Initialisierung des Forecast-Modells auf Basis aktueller Daten durchgeführt [20]. Als Eingabedaten werden die aktuelle Belegung der Prozesse mit Werkstücken, die Prozesszeiten sowie die Restprozesszeiten verwendet. Darüber hinaus werden die Pufferkapazitäten, die Verweildauern und die aktuellen Pufferfüllstände von Verkettungselementen parametrisiert. Die Initialisierung der Verkettungselemente bezieht auch etwaige Restverweildauern der Werkstücke mit ein. Zusätzlich werden die Informationen über zukünftig eintretende Stillstandszeiten bei der Initialisierung berücksichtigt, sodass sämtliche im Lookahead enthaltende Stillstände während des Simulationslaufs des ForecastModells eintreten.

Für die Übertragung der Eingabedaten zwischen den Instanzen wurde auf die Möglichkeit zurückgegriffen, Objektdateien zu erstellen und in einer anderen Instanz wieder einzulesen. Die Kommunikation zwischen den Instanzen wurde durch eine TCP/IP-Schnittstelle realisiert. Dadurch ist es möglich, die Befehle zum Initialisieren, Starten und Zurücksetzen des Forecast-Modells zwischen den Instanzen auszutauschen sowie die Übermittlung der Ergebnisgrößen sicherzustellen.

\section{Simulationsstudie}

Im letzten Kapitel dieses Beitrages werden die Ergebnisse einer durchgeführten Simulationsstudie zur Untersuchung des erzielbaren Mehrwerts einer Priorisierung mit Lookahead vorgestellt. Betrachtet wird eine mehrstufige und mehrdimensionale Prozesskette, die aus vier einfachen sowie zwei redundanten Prozessstufen besteht, wobei alle Prozesse jeweils nur ein Werkstück parallel bearbeiten können (Abbildung 4). Die Prozessstufen sind durch pufferfähige Verkettungselemente verbunden.

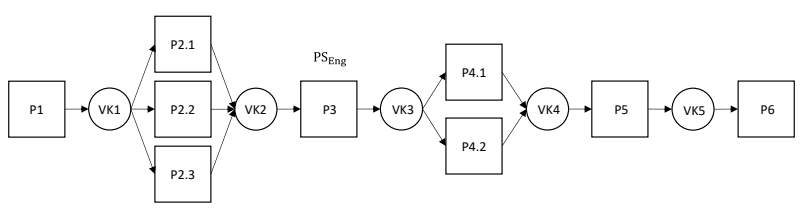

Abbildung 4: Darstellung der mehrdimensionalen Prozesskette

In diesem System ist ein Mitarbeiter ausschließlich für die Behebung von Stillständen zuständig. Die zur Parametrisierung des Modells verwendeten Werte können den Tabellen 1 und 2 entnommen werden. 
Tabelle 1: Eingabedaten der Prozesse

\begin{tabular}{llll}
\hline Prozess & Taktzeit in [s] & $\begin{array}{l}\text { Verfügbarkeit } \\
\text { in [\%] }\end{array}$ & MTTR in [s] \\
\hline P1 & 58 & 93 & 890 \\
\hline P2.1 & 184 & 96 & 640 \\
\hline P2.2 & 186 & 95 & 620 \\
\hline P2.3 & 180 & 97 & 630 \\
\hline P3 & 70 & 95 & 560 \\
\hline P4.1 & 126 & 94 & 580 \\
\hline P4.2 & 130 & 93 & 610 \\
\hline P5 & 65 & 92 & 890 \\
\hline P6 & 59 & 91 & 710 \\
\hline
\end{tabular}

Tabelle 2: Eingabedaten der Verkettungselemente

\begin{tabular}{lll}
\hline $\begin{array}{l}\text { Verkettungsele- } \\
\text { ment }\end{array}$ & $\begin{array}{l}\text { Kapazität in } \\
{[\text { Stück] }}\end{array}$ & $\begin{array}{l}\text { Verweildauer in } \\
{[\mathrm{s}]}\end{array}$ \\
\hline VK1 & 23 & 115 \\
\hline VK2 & 15 & 75 \\
\hline VK3 & 19 & 95 \\
\hline VK4 & 28 & 140 \\
\hline VK5 & 14 & 70 \\
\hline
\end{tabular}

Die Simulationszeit für einen Simulationslauf wurde auf 150 Tage gesetzt, wobei die Einschwingphase zehn Tage beträgt und pro Simulationsexperiment zehn Replikationen simuliert wurden. Während eines Simulationslaufes wird nach jeweils 24 Stunden der erzielte Wert für die Ausbringungsmenge des Fließfertigungssystems innerhalb dieses Zeitraumes ermittelt. Aus diesen Werten wurde dann eine durchschnittliche Ausbringung ermittelt. Folglich beträgt der Stichprobenumfang für die Versuche 1400 .

In Abbildung 5 ist die erzielbare Ausbringung in Abhängigkeit der gewählten Vorgehensweise zur Priorisierung ungeplanter Stillstände dargestellt. Neben der in Kapitel 4 vorgestellten simulationsbasierten Vorgehensweise zu Priorisierung mit Lookahead wurde auch eine FCFS-Strategie umgesetzt. Bei dieser Strategie werden die Stillstände entsprechend ihres Eintrittszeitpunktes priorisiert, wobei zeitlich am frühsten eingetretene Stillstände als Erstes behoben werden. Darüber hinaus wurde eine weitere simulationsbasierte Vorgehensweise implementiert, mit der eine Priorisierung ohne Lookahead möglich ist. Die Vorgehensweise ist dabei überwiegend identisch mit der Vorgehensweise mit Lookahead, wobei sich zwei Änderungen ergeben. Da bei der Vorgehensweise ohne Lookahead keine zukünftig eintretenden geplanten Stillstandszeiten betrachtet werden, müssen diese bei der Initialisierung des Forecast-Modells auch nicht parametrisiert werden. Dadurch ergibt sich ebenfalls ein geändertes Vorgehens zur Ermittlung des Simulationsendzeitpunktes $T_{\text {Ende }}$. Bei dieser Vorgehensweise wird der Simulationsendzeitpunkt statisch auf acht Stunden gesetzt, sodass für jede Behebungsreihenfolge nach acht Stunden Simulationszeit des Forecast-Modells die Durchsatzmenge sowie die Restprozesszeit der Engpassprozessstufe ermittelt wird.

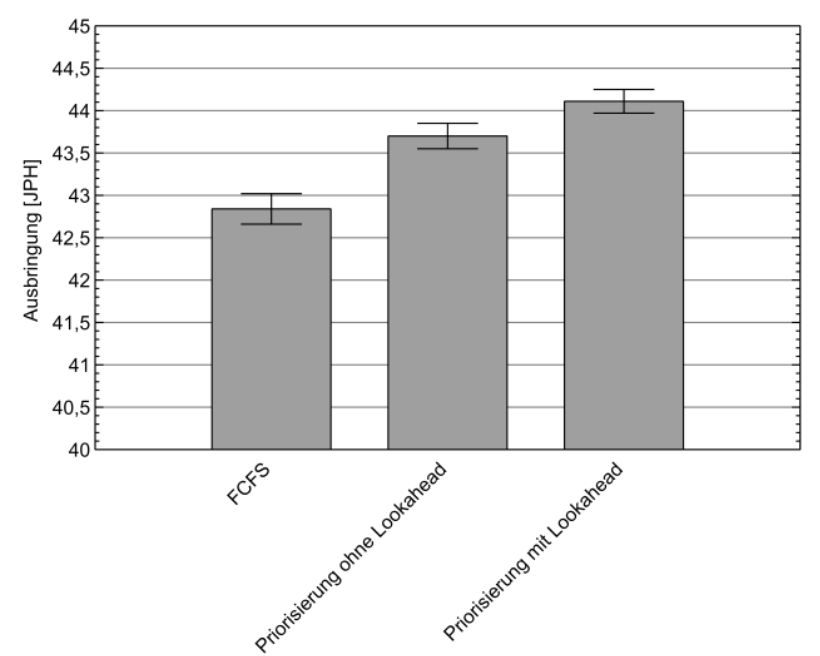

Abbildung 5: Durchschnittlich erzielbare Ausbringung in Abhängigkeit der Priorisierungsmethode

Die Versuchsergebnisse zeigen, dass bereits mit einer Priorisierung ohne Lookahead eine signifikant höhere Ausbringung im Vergleich zu einer FCFS-Strategie erzielt werden kann. Dabei liegt die durchschnittliche Ausbringung einer Priorisierung ohne Lookahead bei 43,7 \pm 0,15 JPH (95 \% Konfidenzintervall), was einem Mehrwert im Vergleich zur FCFS-Strategie von 2,01 \% entspricht. Durch Berücksichtigung zukünftig eintretender geplanter Stillstandszeiten in Form eines Lookahead kann darüber hinaus mit 44,1 \pm 0,14 JPH (95 \% Konfidenzintervall) eine signifikant höhere Ausbringung generiert werden. Der Mehrwert gegenüber der FCFS-Strategie beträgt dabei 2,96 \%, wobei die Ermittlung einer Priorisierungsreihenfolge nur in $24 \%$ aller Entscheidungen, bei denen der Produktionsmitarbeiter zu einem oder 
mehreren Stillständen zugeteilt werden musste, angewendet wurde. Somit liegt bei 76 \% der Entscheidungen nur ein Stillstand an, wobei in diesen Fällen der Mitarbeiter direkt zugeordnet wird. Der direkte Vergleich einer Priorisierung mit Lookahead zu einer Priorisierung ohne Lookahead zeigt schließlich, dass eine Priorisierung mit Lookahead zu einem signifikanten Mehrwert von 0,92 \% führt. Dieser Mehrwert resultiert aus der Möglichkeit, durch die Wahl der Priorisierungsreihenfolge die Auswirkung geplanter Stillstandszeiten proaktiv zu beeinflussen. Da die Stillstandsdauer der statischen Engpassprozessstufe oder auch einer Prozessstufe, für die zukünftig ein Stillstand eingeplant ist, abhängig von der Priorisierungsreihenfolge ist, kann der Durchsatz dieser Prozessstufe und somit auch die Anzahl an Werkstücken, die sich zum Eintrittszeitpunkt eines geplanten Stillstandes zwischen der betroffenen Prozessstufe und der statischen Engpassprozessstufe befinden, durch die Priorisierung beeinflusst werden. Die freie Pufferkapazität in diesem Bereich entscheidet letztendlich über die Dauer der latenten Phase, d. h. der Zeitspanne, in der sich die Auswirkung eines Stillstandes noch nicht auf die Leistung des gesamten Fließfertigungssystems auswirkt, sodass sich folglich mit der Priorisierungsreihenfolge auch die Auswirkung geplanter Stillstände beeinflussen lässt.

Der Lookahead wurde hier als Time-Lookahead definiert. Dabei sind sämtliche Stillstandszeiten innerhalb der nächsten acht Stunden Simulationszeit, bezogen auf den jeweiligen Entscheidungszeitpunkt $t_{d}$, bekannt und wurden dementsprechend bei der Ermittlung der Priorisierungsreihenfolge berücksichtigt. Damit stellt der hier ermittelte Wert für die mit einer Priorisierung mit Lookahead erzielbare Ausbringung eine obere Grenze dar, da alle Stillstandszeiten während dieses Zeitraumes bekannt sind. In der Praxis kann allerdings nicht davon ausgegangen werden, dass sämtliche zukünftig eintretende Stillstandszeiten zum jeweiligen Entscheidungszeitpunkt bekannt sind. Deswegen wurde in einem weiteren Experiment untersucht, wie der Mehrwert einer Priorisierung mit Lookahead beeinflusst wird, wenn neben den geplanten und im Lookahead enthaltenen Stillständen auch ungeplante Stillstände, d. h. zum Entscheidungszeitpunkt nicht bekannte und damit auch nicht in die Priorisierungsentscheidung mit einbezogene Stillstände, eintreten. In Abbildung 6 ist der Mehrwert einer Priorisierung mit Lookahead in Abhängigkeit des Anteils geplanter Stillstände an der Gesamtanzahl eintretender Stillstände dargestellt.

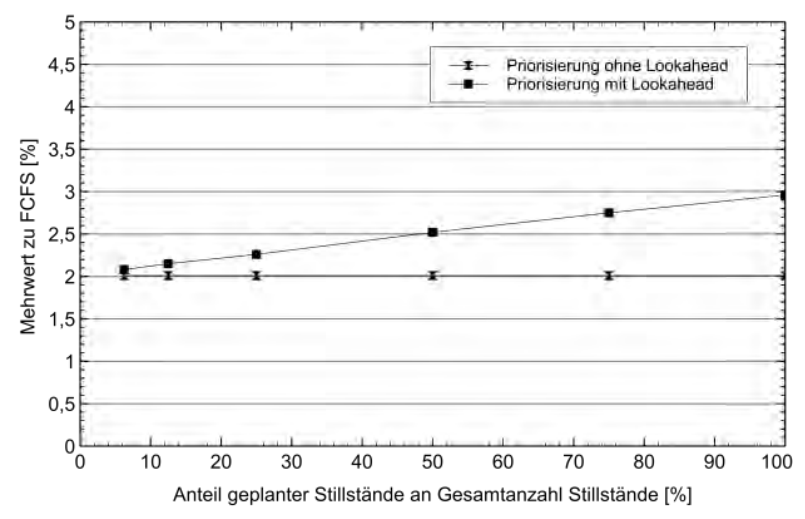

Abbildung 6: Einfluss des Anteils geplanter Stillstände auf den Mehrwert einer Priorisierung im Vergleich zur FCFS-Strategie

Es zeigt sich, dass der Mehrwert einer Priorisierung mit Lookahead mit sinkendem Anteil geplanter Stillstände abnimmt, wobei der Mehrwert stets größer als der Mehrwert einer Priorisierung ohne Lookahead bleibt. Weitere Untersuchungen einzelner Priorisierungsentscheidungen haben hier gezeigt, dass mit abnehmendem Anteil geplanter Stillstände eine gezielte Beeinflussung der verfügbaren Pufferkompensation zum Eintrittszeitpunkt eines geplanten Stillstandes nicht mehr möglich ist. Die verfügbare Pufferkompensation wird vielmehr durch das Auftreten der ungeplanten und nicht im Lookahead enthaltenen Stillstände beeinflusst, sodass eine Priorisierung mit Lookahead bei einem geringen Anteil geplanter Stillstände zu keinem signifikanten Mehrwert gegenüber einer Priorisierung ohne Lookahead führt.

\section{Zusammenfassung und Ausblick}

In diesem Beitrag wurde der Mehrwert einer Priorisierung von Maschinenstillständen unter Berücksichtigung zukünftig eintretender geplanter Stillstandszeiten untersucht. Dazu wurde die Priorisierung als Online-Optimierungsproblem mit Lookahead aufgefasst und eine simulationsbasierte Vorgehensweise vorgestellt, mit der geplante Stillstandszeiten in die Ermittlung einer Priorisierungsreihenfolge mit einbezogen werden können. Durch die Implementierung dieser Vorgehensweise als Entscheidungsunterstützungssystem in eine Simulationsumgebung, konnte schließlich am Beispiel eines mehrdimensionalen Fließfertigungssystems ein signifikanter Mehrwert einer Priorisierung mit Lookahead im Vergleich zu einer Priorisierung ohne Lookahead aufgezeigt 
werden, wobei der erzielbare Mehrwert von dem Anteil geplanter und damit im Lookahead berücksichtigter Stillstände abhängig ist.

Im weiteren Verlauf der Forschungsarbeit sollen weitere Einflussfaktoren auf den Mehrwert einer Priorisierung mit Lookahead identifiziert werden. Darauf aufbauend wird untersucht, unter welchen Randbedingungen eine Priorisierung mit Lookahead zu einem signifikanten Mehrwert führt. Zudem soll die simulationsbasierte Vorgehensweise zur Priorisierung mit Lookahead auch in einer praxisnahen Umgebung validiert werden. Dazu wird der erzielbare Mehrwert in Kooperation mit der Mercedes-Benz AG an einer komplexen Kurbelgehäusefertigungslinie überprüft.

\section{Literatur}

[1] Kröning, S.; Denkena, B.: Dynamic scheduling of maintenance measures in complex production systems. Journal of Manufacturing Science and Technology 6 (2013) 4, S. 292-300, S. 292

[2] Wedel, M.: Effektive Priorisierung bei reaktiven Instandhaltungsmaßnahmen zur Steigerung der Ausbringung von komplexen Transferstraßen am Beispiel der Automobilindustrie. Aachen: Shaker 2016, S. 2

[3] Zhai, S.; Reinhart, G.: Predictive Maintenance als Wegbereiter für die instandhaltungsgerechte Produktionssteuerung. Zeitschrift für wirtschaftlichen Fabrikbetrieb (ZWF) 113 (2018) 5, S. 298-301, S. 299

[4] Hegemann, M.; Nickel, S.: Einfluss von zuverlässig prognostizierten Stillstandzeiten auf die simulationsbasierte Priorisierung von Maschinenstillständen in komplexen Produktionssystemen. In: ASIM Fachtagung Simulation in Produktion und Logistik 2019. Chemnitz: 18.-20.09.2019

[5] Guo, W.; Jin, J.; Hu, S. J.: Allocation of maintenance resources in mixed model assembly systems. In. Journal of Manufacturing Systems 32 (2013), S. 473-479

[6] Bengtsson, M.: Classification of Machine Equipment. In: Conference on Maintenance Performance Measurement and Management. Lulea (SWE), 2011, S. 1-5

[7] Gupta, S.; Bhattacharya, J.: Cost.effiective importance measure: A new approach for resource prioritization in a production plant. In: International Journal of Quality and Reliably Management 30 (2013) 4, S. 379-386

[8] Gopalakrishnan, M.; Skoogh, A.; Laroque, C.: Simulation-based Planning of Maintenance Activities in the Automotive Industry. In: Proceedings of the 2013 Winter Simulation Conference. Washington D. C. (USA), 2013, S. $2610-2621$

[9] Guner, H. U.; Chinnam, R.; Murat, A.: Simulation platform for anticipative plant-level maintenance decision support system. In: International Journal of Production Research (2015), S. 1-19
[10] Gopalakrishnan, M.; Skoogh, A.; Laroque, C.: Simulation-based Planning of Maintenance Activities by a Shifting Priority Method. In: Proceedings of the 2014 Winter Simulation Conference. Savannah Georgia. (USA), 2014, S. 2168-2179

[11] Langer, R.; Li, J.; Biller, S.; Chang, Q.; Huang, N.; Xiao, G.: Simulation study of a bottleneck-based dispatching policy for a maintenance workforce. In: International Journal of Production Research 48 (2010) 6, S. 17451763

[12] Li, L.; Chang, Q.; Ni, J.; Biller, S.: Real time production improvement through bottleneck control. In: International Journal of Production Research. 47 (2009a) 21, S. 6145-6158

[13] Li, L.; Ni, J.: Short-term decision support system for maintenance task prioritization. In: International Journal of Production Economics 121 (2009), S. 195-202

[14] Yang, Z.; Chang, Q.; Djurdjanovic, D.; Ni, J.; Lee, J.: Maintenance Priority Assignment Utilizing On-line Production Information. In. International Journal of Manufacturing Science and Engineering 129 (2007), S. 435446

[15] Ni, J.; Jin, X.: Decision support systems for effective maintenance operations. In: CIRP Annals - Manufacturing Technology 61 (2012), S. 411-414

[16] Subramaniyan, M.; Skoogh, A.; Gopalakrishnan, M.; Salomonsson, H.; Hanna, A.; Lämkull, D.: An algorithm for data-driven shifting bottleneck detection. In: Cogent Engineering 3 (2016), S. 1-19

[17] Subramaniyan, M.; Skoogh, A.; Salomonsson, H.; Bangalore, P.: A data-driven algorithm to predict throughput bottlenecks in a production system based on active periods of machines. In: Computers and Industrial Engineering 125 (2018), S. 533-544

[18] Dunke, F.; Necil, J.; Nickel, S.: Online-Optimierung und Simulation in der Logistik. In: Lübbecke, M.; Weiler, A.; Werners, B.: Zukunftsperspektiven des Operations Research. Wiesbaden. Springer Gabler: 2014, S. 33-47

[19] Dunke, F.; Nickel, S.: A general modeling approach to online optimization with lookahead. In: Omega 63 (2016), S. 134-153

[20] Hanisch, A.; Schulze, T.: Initialization of Online Simulation Models. In: Proceedings of the 2005 Winter Simulation Conference. Orlando (USA), 2005, S. 1795-1803 